\title{
Evaluation of reliability bounds by set covering models
}

\author{
M.V. Koutras ${ }^{\mathrm{a}, *}$, S. Tsitmidelis ${ }^{\mathrm{b}}$, V. Zissimopoulos ${ }^{\mathrm{c}}$ \\ ${ }^{a}$ Department of Statistics and Actuarial Science, University of Piraeus, 80 Karaoli \& Dimitriou Str, \\ Piraeus 18534, Greece \\ ${ }^{\mathrm{b}}$ Department of Applied Sciences, T.E.I. of Chalkis, Psahna Evoias 34400, Greece \\ ${ }^{\mathrm{c}}$ Department of Informatics and Telecommunications, University of Athens, Panepistimiopolis 15784, Greece
}

Received February 2002; received in revised form September 2002

\begin{abstract}
In the present article, we deal with the problem of developing a systematic procedure for evaluating the general reliability bounds developed recently by Fu and Koutras (Statist. Probab. Lett. 22 (1995) 137). More specifically, we prove that, the identification of the optimal bounds can be achieved by transforming the set-theoretic and probabilistic conditions associated with the bounds to an equivalent set covering problem (SC). As a consequence, available solution algorithms for the SC (exact or heuristic) can be exploited to derive very tough approximation intervals for a general system's reliability at very competitive computer times as compared to the respective exact reliability evaluation algorithms.
\end{abstract}

(c) 2003 Elsevier Science B.V. All rights reserved.

Keywords: Reliability bounds; Cut sets; Path sets; Set covering problem; Genetic algorithm

\section{Introduction}

In the present article we consider binary reliability structures, that is systems consisting of components that can only be in one of two states, operational (working, on, up) or failed (not working, off, down). Let $I=\{1,2, \ldots, n\}$ be the set of all components of the system and denote by $z_{i}, i \in I$ the state of component $i$ at a fixed instance, that is

$$
z_{i}= \begin{cases}1 & \text { if component } i \text { is working } \\ 0 & \text { if component } i \text { is not working. }\end{cases}
$$

\footnotetext{
${ }^{*}$ Corresponding author. Tel.: +30-1-4142393; fax: +30-1-4142340.

E-mail address: mkoutras@unipi.gr (M.V. Koutras).
} 
The state of the system is completely determined by the state of its components, and the dependence of the system state on the element states is usually expressed through the so-called structure function $\varphi\left(z_{1}, z_{2}, \ldots, z_{n}\right)$ which takes on the value 1 if the system is up and the value 0 if the system is down. An alternative method to describe a reliability structure is through the family of minimal cut sets

$$
\boldsymbol{C}=\left\{C_{1}, C_{2}, C_{3}, \ldots, C_{N}\right\}
$$

or the family of minimal path sets.

$$
\boldsymbol{P}=\left\{P_{1}, P_{2}, P_{3}, \ldots, P_{M}\right\}
$$

A minimal cut set is a minimal set of components whose failure results in system's failure. Likewise a minimal path set is a minimal set of components whose functioning ensures the functioning of the system. It can be proved (see e.g. Barlow and Proschan, 1975) that any coherent reliability system can be represented as a series connection of parallel structures corresponding to the minimal cut sets or as a parallel connection of series structures corresponding to the minimal path sets. These facts are mathematically expressed by the following two formulae:

$$
\varphi\left(z_{1}, z_{2}, \ldots, z_{n}\right)=\prod_{j=1}^{N}\left(1-\prod_{i \in C_{j}}\left(1-z_{i}\right)\right)=1-\prod_{j=1}^{M}\left(1-\prod_{i \in P_{j}} z_{i}\right) .
$$

The reliability of the system can be written as

$$
R_{n}=E\left[\varphi\left(Z_{1}, Z_{2}, \ldots, Z_{n}\right)\right],
$$

where $Z_{i}$ is a binary random variable associated with the $i$ th component's state at a prespecified instance. For the evaluation of $R_{n}$ by the aid of the last expression one has to reduce first $\varphi\left(z_{1}, z_{2}, \ldots, z_{n}\right)$ in a sum of products of $z_{i}$ and then apply the mean value operator. When the number of minimal cut/path sets of the system is large this approach may lead to computationally demanding algorithms. In such cases, the development of manageable lower and upper bounds offering satisfactory estimates of system's reliability may be proved quite useful.

From now on we assume that the components of the system work independently, and denote by $p_{i}=E\left(z_{i}\right), q_{i}=1-p_{i}, i \in \boldsymbol{I}$ their survival and failure probabilities, respectively. One of the earliest approaches towards constructing simple reliability bounds for coherent structures with independent components, should be attributed to Esary and Proschan (1963) who proved that

$$
\prod_{j=1}^{N}\left(1-\prod_{i \in C_{j}}\left(1-p_{i}\right)\right) \leqslant R_{n} \leqslant 1-\prod_{j=1}^{M}\left(1-\prod_{i \in P_{j}} p_{i}\right) .
$$

The lower bound

$$
L_{\mathrm{EP}}=\prod_{j=1}^{N}\left(1-\prod_{i \in C_{j}}\left(1-p_{i}\right)\right)
$$

can be interpreted as the reliability of a fictitious system in which each minimal cut set of the original system has been used to construct a parallel subsystem and all these subsystems have been 
independently replicated and connected in series. In a similar vain, the upper bound

$$
U_{\mathrm{EP}}=1-\prod_{j=1}^{M}\left(1-\prod_{i \in P_{j}} p_{i}\right)
$$

is the reliability of another fictitious system in which each minimal path set of the original system has been used to construct a series subsystem and all these subsystems have been independently replicated and connected in parallel.

Due to their simplicity, the aforementioned bounds have by now been incorporated into many graduate and post-graduate textbooks; see for example Barlow and Proschan (1975) or Ross (1985). Unfortunately, the Esary and Proschan bounds cannot be effectively used for approximating system reliability from both sides (above and below); as numerical experimentation indicates, $L_{\mathrm{EP}}$ yields good approximations for high reliability structures while $U_{\mathrm{EP}}$ performs well only for low reliability structures. Motivated by this observation, $\mathrm{Fu}$ and Koutras (1995) established recently two classes of reliability bounds which can be effectively coupled with the Esary and Proschan bounds to construct very tight intervals estimates; see also Boutsikas and Koutras (2000) for a further generalization. Their bounds have the form

$$
L_{\mathrm{FK}}=1-\prod_{j=1}^{M}\left[1-\left(\prod_{i \in K_{j}} q_{i}\right)\left(\prod_{i \in P_{j}} p_{i}\right)\right] \leqslant R_{n} \leqslant \prod_{j=1}^{N}\left[1-\left(\prod_{i \in L_{j}} p_{i}\right)\left(\prod_{i \in C_{j}} q_{i}\right)\right]=U_{\mathrm{FK}},
$$

where $L_{j}, K_{j} \subseteq I$ are appropriate index sets satisfying specific conditions. Although there always exist such index sets, the authors have not provided any standard procedure leading to the determination of them.

In the present article, we prove that the optimal choices of $L_{j}, K_{j}$ can be acquired by solving non-unicost set covering problems (SC) that is, problems of the form

$$
\begin{array}{ll}
\text { Minimize } & \sum_{t \in T} c_{t} x_{t} \\
\text { Subject to } & \sum_{t \in T} \alpha_{v t} x_{t} \geqslant 1 \quad \text { for all } v \in V,
\end{array}
$$

where $A=\left(\alpha_{v t}\right)$ is a $|V| \times|T|$ matrix with entries 0 or $1\left(\alpha_{v t} \in\{0,1\}\right), V$ is the set of rows to be covered, $T$ is the set of columns for covering the rows and $c_{t}$ are cost coefficients determined by the survival probabilities of the components. Therefore, solution algorithms for the SC can be applied to evaluate the bounds $L_{\mathrm{FK}}$ and $U_{\mathrm{FK}}$. Since the SC is well known to be NP-complete (see e.g. Lund and Yannakakis, 1994; Johnson, 1974; Lovász, 1975) a great research effort has been invested in establishing effective algorithms for solving problems of practical size, Balas (1980), Beasley (1990), Fischer and Kedia (1990), Afif et al. (1995).

A variety of heuristic solution methods have also been developed for SC's. For a review on them the interested reader may consult Paschos (1997). In a recent paper, Beasley and Chu (1996) introduced a genetic algorithm-based heuristic for set covering problems, which is capable of producing high quality solutions. In the present article, we employed Beasley and Chu's algorithm to test, numerically, the effectiveness of the suggested procedure in establishing tight interval estimates for 
the unknown system reliability. Moreover, a variation of this algorithm was also put to test. Our extensive numerical experimentation revealed that this approach can be efficiently applied even for large reliability structures.

\section{Cut set-based reliability bounds}

In this section, we assume that the family $\boldsymbol{C}$ of minimal cut sets of a coherent structure is given by (1.1) and that the components of the structure work independently. Then the Esary and Proschan lower bound (1.3) can be easily evaluated. On the other hand, the $\mathrm{Fu}$ and Koutras upper bound (1.5) can be computed, provided that the index sets $L_{j}, j=1,2, \ldots N$ have been identified. A direct comparison of $L_{\mathrm{EP}}$ and $U_{\mathrm{FK}}$ reveals that, for systems involving high reliability components, the discrepancy between the lower and upper bound is expected to be quite small. Moreover, for specific $p_{i}$ 's, the best choice for $U_{\mathrm{FK}}$ is the one that maximizes the products $\prod_{i \in L_{j}} p_{i}$, or equivalently, the one that maximizes

$$
-\ln \prod_{i \in L_{j}} p_{i}=\sum_{i \in L_{j}}\left(-\ln p_{i}\right) .
$$

We recall that the index sets $L_{j}$ in $\mathrm{Fu}$ and Koutras upper bounds $U_{\mathrm{FK}}$ are not determined in a unique way. In order to arrive at a valid selection of $L_{j}$, we introduce first the auxiliary index sets

$$
\begin{aligned}
& L_{1}^{*}=\varnothing, \\
& L_{j}^{*}=\left\{i: C_{i} \cap C_{j} \neq \varnothing, 1 \leqslant i \leqslant j\right\}, j=2,3, \ldots, N
\end{aligned}
$$

and for each nonempty $L_{j}^{*}$ we construct another index set $L_{j}$ satisfying the conditions

$$
\begin{aligned}
& L_{j} \cap C_{j}=\varnothing, \\
& L_{j} \cap C_{i} \neq \varnothing \quad \text { for every } i \in L_{j}^{*} .
\end{aligned}
$$

If $L_{j}^{*}=\varnothing$ we set $L_{j}=\varnothing$. It is evident that, for any coherent system, there always exist $L_{j} \subseteq \boldsymbol{I}$ satisfying (2.3), (2.4); for example,

$$
L_{1}=\varnothing, \quad L_{j}=\bigcup_{i=1}^{j-1}\left(C_{i} \cap C_{j}^{\prime}\right), \quad j=2, \ldots, N
$$

consist always a valid choice of $L_{j}\left(C_{j}^{\prime}\right.$ stands for the set theoretic complement of $C_{j}$ with respect to $I$ ). The next question to be answered is, how could one technically arrive at optimal choices of $L_{j}$, that is the ones that minimize (2.1)? For small systems or systems with special structure of the cut sets, one may manually identify the optimal choices of $L_{j}$ by exploiting conditions (2.3) and (2.4). However in the general case, this task becomes quite involved and this is why we need an alternative modeling of the problem at hand so that standard computer procedures (instead of set operations ) can be applied. To start with, let us fix $j(j \geqslant 2)$ and observe first that condition (2.3) implies that $L_{j} \subseteq C_{j}^{\prime}$. If we set

$$
C_{j}^{\prime}=\left\{j_{1}, j_{2}, \ldots, j_{n-\left|C_{j}\right|}\right\}
$$


it is evident that $L_{j}$ is(uniquely) determined by a binary vector

$$
\boldsymbol{x}=\left(x_{j_{1}}, x_{j_{2}}, \ldots, x_{\left.j_{n-\left|c_{j}\right|}\right)}\right)
$$

whose entries $x_{j_{s}} \in\{0,1\}$ are defined by

$$
\boldsymbol{x}_{j_{s}}= \begin{cases}1 & \text { if component } j_{s} \text { belongs to } L_{j}, \\ 0 & \text { if component } j_{s} \text { does not belong to } L_{j} .\end{cases}
$$

On the other hand, on introducing a binary matrix $A=\left(\alpha_{i k}\right)_{N x n}$ by

$$
\alpha_{i k}= \begin{cases}1 & \text { if component } k \text { belongs to minimal cut set } C_{i}, \\ 0 & \text { if component } k \text { does not belong to minimal cut set } C_{i} .\end{cases}
$$

for $i=1,2, \ldots, N$ and $k=1,2, \ldots, n$ condition (2.4) takes on the following equivalent form:

$$
\sum_{k \in C_{j}^{\prime}} \alpha_{i k} x_{k} \geqslant 1 \text { for all } i \in L_{j}^{*} \text {. }
$$

Any feasible solution $\boldsymbol{x}=\left(x_{j_{1}}, x_{j_{2}}, \ldots, x_{j_{n-\mid C_{j}} \mid}\right)$ of the above system of $\left|L_{j}^{*}\right|$ inequalities, gives rise to a valid choice of $L_{j}$, namely

$$
L_{j}=\left\{i \in C_{j}^{\prime}: x_{i}=1\right\} \text {. }
$$

\section{The algorithm}

According to the analysis performed at the beginning of the present paragraph, the optimum choice of $L_{j}$ (in the sense that the respective upper bound $U_{\mathrm{FK}}$ shifts as close to the lower bound $L_{\mathrm{EP}}$ as possible) is achieved by minimizing the sum (2.1), or equivalently

$$
f(x)=\sum_{k \in C_{j}^{\prime}}\left(-\ln p_{k}\right) x_{k}=\sum_{k \in C_{j}^{\prime}} c_{k} x_{k}
$$

where

$$
c_{k}=-\ln p_{k}, \quad k=1,2, \ldots, n .
$$

Therefore, the following algorithm can be exploited for deducing the Esary and Proschan lower bounds $L_{\mathrm{EP}}$ and the bound $U_{\mathrm{FK}}$ for a coherent structure whose family of minimal cut sets (1.1) has been given.

Step 1: Set $L_{\mathrm{EP}}=U_{\mathrm{FK}}=1$ (initial values) and evaluate $\alpha_{i k}$ and $c_{k}$ by (2.5) and(2.6), respectively.

Step 2: Repeat steps 3-6 for $j=1,2, \ldots, N$.

Step 3: Set $L_{\mathrm{EP}}=L_{\mathrm{EP}} *\left(1-\prod_{i \in C_{j}} q_{i}\right)$.

Step 4: Compute the auxiliary index set $L_{j}^{*}$ defined by (2.2). If $L_{j}^{*}=\varnothing$ then set $U_{\mathrm{FK}}=U_{\mathrm{FK}} *(1-$ $\prod_{i \in C_{j}} q_{i}$ ) and return to step 3 (for the next $j$ ). If $L_{j}^{*} \neq \varnothing$ then perform steps 5-6.

Step 5: Solve the weighted set covering problem:

Minimize $\sum_{t \in T} c_{t} x_{t}$

Subject to $\sum_{t \in T} \alpha_{v t} x_{t} \geqslant 1 \quad$ for all $v \in V, x_{t} \in\{0,1\}$ for all $t \in T$, 
where

$V=L_{j}^{*}$ the set of rows to be covered,

$T=C_{j}^{\prime}$ the set of columns for covering the rows.

Step 6. If $\left\{x_{t}, t \in T\right\}$ is the solution obtained in step 5, compute $L_{j}$ by

$$
L_{j}=\left\{t \in T: x_{t}=1\right\}
$$

and set

$$
U_{\mathrm{FK}}=U_{\mathrm{FK}}\left[\prod_{j=1}^{N}\left[1-\left(\prod_{i \in L_{j}} p_{i}\right)\left(\prod_{i \in C_{j}} q_{i}\right)\right]\right] .
$$

Then return to step 3 (for the next $j$ ).

After having completed steps 1-6, the variable $L_{\mathrm{EP}}$ and $U_{\mathrm{FK}}$ contain the Esary and Proschan lower bound and the optimal $\mathrm{Fu}$ and Koutras upper bound for system's reliability, respectively.

It is worth mentioning that the SC described in step 5 involves $\left|L_{j}^{*}\right|$ rows and at most $\left|C_{j}^{\prime}\right|=n-\left|C_{j}\right|$ columns. As a matter of fact the number of columns is strictly less than $n-\left|C_{j}\right|$ if for some $t$ 's it holds true that $\alpha_{v t}=0$ for all $v \in V=L_{j}^{*}$. Another pertinent remark here is that, in the iid case (identical components) $p_{i}=p, i=1,2, \ldots, n$ the $\mathrm{SC}$ mentioned in step 5 reduces to the unicost (unweighted) problem i.e. the objective function can be replaced by $f(x)=\sum x_{t}$.

As an illustration, let us consider the following pipeline system:

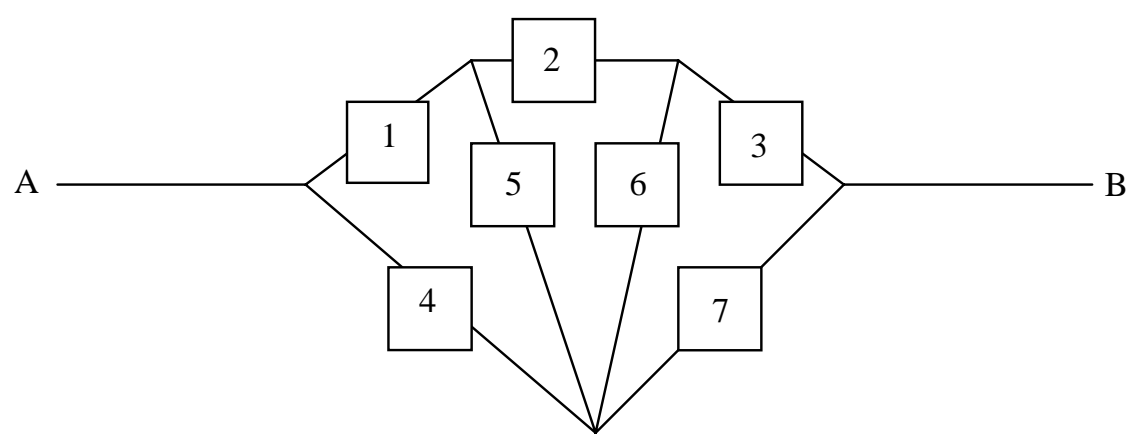

Arcs 1-7 represent seven pump stations used for transferring water from points $\mathrm{A}$ to $\mathrm{B}$. The set of components is $\boldsymbol{I}=\{1,2, \ldots, 7\}$ and after performing an elementary analysis of the structure we may readily verify that the family $C$ of minimal cut sets contains $N=5$ elements, namely

$$
C_{1}=\{1,4\}, C_{2}=\{1,5,6,7\}, C_{3}=\{2,4,5\}, C_{4}=\{3,4,5,6\}, C_{5}=\{3,7\}
$$

(the arrangement of $C_{j}$ 's was done according to the minimum component label contained in each one). 
Matrix $A$ takes on the form

\begin{tabular}{llllclll} 
Cut sets & \multicolumn{7}{c}{ Components } \\
\cline { 2 - 7 } & 1 & 2 & 3 & 4 & 5 & 6 & 7 \\
\hline$C_{1}$ & 1 & 0 & 0 & 1 & 0 & 0 & 0 \\
$C_{2}$ & 1 & 0 & 0 & 0 & 1 & 1 & 1 \\
$C_{3}$ & 0 & 1 & 0 & 1 & 1 & 0 & 0 \\
$C_{4}$ & 0 & 0 & 1 & 1 & 1 & 1 & 0 \\
$C_{5}$ & 0 & 0 & 1 & 0 & 0 & 0 & 1 \\
\hline
\end{tabular}

while $L_{j}^{*}, j=1,2,3,4,5$ are easily checked to be

$$
L_{1}^{*}=\varnothing, L_{2}^{*}=\{1\}, L_{3}^{*}=\{1,2\}, L_{4}^{*}=\{1,2,3\}, L_{5}^{*}=\{2,4\} .
$$

Manifestly $L_{1}=\varnothing$. In order to compute $L_{2}$, we consider

$$
V=L_{2}^{*}=\{1\}, T=C_{2}^{\prime}=\{1,5,6,7\}^{\prime}=\{2,3,4\}
$$

and write down the system of inequalities deduced by singling out the first row and columns 2-4 of matrix $\boldsymbol{A}$. This results to the conditions

$$
\begin{aligned}
& x_{4} \geqslant 1, \\
& x_{4} \in\{0,1\},
\end{aligned}
$$

which yield to $x_{4}=1$; hence $L_{2}=\{4\}$.

Working in a similar fashion we may effortlessly verify that $L_{3}$ and $L_{4}$ are uniquely determined as $L_{3}=\{1\}$ and $L_{4}=\{1,2\}$, respectively. Finally the SC associated to $L_{5}$ makes use of $V=L_{5}^{*}=$ $\{2,4\}, T=C_{5}^{\prime}=\{3,7\}^{\prime}=\{1,2,4,5,6\}$ and the respective inequalities take on the form (after deleting the column corresponding to component 2 which has all its entries 0 )

$$
\begin{aligned}
& x_{1}+x_{5}+x_{6} \geqslant 1, \\
& x_{4}+x_{5}+x_{6} \geqslant 1, \\
& x_{1}, x_{4}, x_{5}, x_{6} \in\{0,1\} .
\end{aligned}
$$

The set of feasible solutions of the above system contains 14 elements and the optimal choice of $L_{5}$ will be carried out by minimizing

$$
f(x)=c_{1} x_{1}+c_{4} x_{4}+c_{5} x_{5}+c_{6} x_{6},
$$

where $c_{i}=-\ln p_{i}, i=1,4,5,6$. It can be shown, by carrying out an elementary analysis on the system of inequalities, that the optimal choice depends on the relative magnitude of $p_{5}, p_{6}$ and $p_{1} p_{4}$; more specifically:

- If $\max \left(p_{5}, p_{6}, p_{1} p_{4}\right)=p_{6}$ then the optimal solution is $\left(x_{1}, x_{4}, x_{5}, x_{6}\right)=(0,0,0,1)$ and the optimal choice for $L_{5}$ is given by $L_{5}=\{6\}$. 
- If $\max \left(p_{5}, p_{6}, p_{1} p_{4}\right)=p_{5}$ then the optimal solution is $\left(x_{1}, x_{4}, x_{5}, x_{6}\right)=(0,0,1,0)$ and the optimal choice for $L_{5}$ is given by $L_{5}=\{5\}$.

- If $\max \left(p_{5}, p_{6}, p_{1} p_{4}\right)=p_{1} p_{4}$ then the optimal solution is $\left(x_{1}, x_{4}, x_{5}, x_{6}\right)=(1,1,0,0)$ and the optimal choice for $L_{5}$ is given by $L_{5}=\{1,4\}$.

Therefore, the Esary and Proschan lower bound and the Fu and Koutras upper bound are expressed as

$$
\begin{aligned}
& L_{\mathrm{EP}}=\left(1-q_{1} q_{4}\right)\left(1-q_{1} q_{5} q_{6} q_{7}\right)\left(1-q_{2} q_{4} q_{5}\right)\left(1-q_{3} q_{4} q_{5} q_{6}\right)\left(1-q_{3} q_{7}\right), \\
& U_{\mathrm{FK}}=\left(1-q_{1} q_{4}\right)\left(1-p_{4} q_{1} q_{5} q_{6} q_{7}\right)\left(1-p_{1} q_{2} q_{4} q_{5}\right)\left(1-p_{1} p_{2} q_{3} q_{4} q_{5} q_{6}\right)\left(1-r q_{3} q_{7}\right),
\end{aligned}
$$

where $r=p_{6}$ or $p_{5}$ or $p_{1} p_{4}$. It is worth mentioning that any of the three available choices of $r$ leads to an upper bound for system's reliability; however the optimal choice described earlier will result in the best (smaller) upper bound. In the iid case, $p_{i}=p, q_{i}=q i=1,2, \ldots, 7$, the optimal choice for $L_{5}$ is $L_{5}=\{5\}$ or $L_{5}=\{6\}$ and the aforementioned bounds reduce to

$$
\begin{aligned}
& L_{\mathrm{EP}}=\left(1-q^{2}\right)^{2}\left(1-q^{3}\right)\left(1-q^{4}\right)^{2}, \\
& U_{\mathrm{FK}}=\left(1-q^{2}\right)\left(1-p q^{4}\right)\left(1-p q^{3}\right)\left(1-p^{2} q^{4}\right)\left(1-p q^{2}\right) .
\end{aligned}
$$

From expressions (2.7), (2.8) it is clear that, should the components of the system be highly reliable (that is if $\min p_{i}$ or $p$ (in the iid case) is close to 1 ), $U_{\mathrm{FK}}$ will approach $L_{\mathrm{EP}}$ thereof obtaining a very tight interval estimate of system's reliability. On the other hand, if the components are unreliable (that is, if $\min q_{i}$ or $q$ (in the iid case) is close to 1), $U_{\mathrm{FK}}$ will depart from $L_{\mathrm{EP}}$ and the resulting interval may be useless. In this situation it is preferable to work with the path set based bounds $L_{\mathrm{FK}}$ and $U_{\mathrm{EP}}$ as described in (1.4) and (1.5). Given the family $\boldsymbol{P}$ (cf. (1.2)) of minimal path sets of the structure, the Esary and Proschan upper bound $U_{\mathrm{EP}}$ can be easily calculated by (1.4). In order to evaluate the $\mathrm{Fu}$ and Koutras lower bound $L_{\mathrm{FK}}$ we first introduce the auxiliary index sets

$$
\begin{aligned}
& K_{1}^{*}=\varnothing, \\
& K_{j}^{*}=\left\{i: P_{i} \cap P_{j} \neq \varnothing, \quad 1 \leqslant i \leqslant j\right\}, j=2,3, \ldots, M
\end{aligned}
$$

and for each nonempty $K_{j}^{*}$ we construct another index set $K_{j}$ satisfying the conditions

$$
\begin{aligned}
& K_{j} \cap P_{j}=\varnothing, \\
& K_{j} \cap P_{i} \neq \varnothing \quad \text { for every } i \in K_{j}^{*} .
\end{aligned}
$$

For $K_{j}^{*}=\varnothing$ we set $K_{j}=\varnothing$. The construction of the (nonempty) index sets $K_{j}$ can be carried out by transforming the problem to the following SCP, the derivation following an exact parallel to that used for cut sets. Let $A=\left(\alpha_{i k}\right)_{M x n}$ be a binary matrix defined by

$$
\alpha_{i k}= \begin{cases}1 & \text { if component } k \text { belongs to minimal path set } P_{i}, \\ 0 & \text { if component } k \text { does not belong to minimal path set } P_{i}\end{cases}
$$


and set $c_{k}=-\ln q_{k}, k=1,2, \ldots, n$. Then the optimal choice for $K_{j}$ is provided by

$$
K_{j}=\left\{t \in T: x_{t}=1\right\}
$$

where $\left\{x_{t}, t \in T\right\}$ is the solution of the weighted SC

$$
\begin{array}{ll}
\text { minimize } & \sum_{t \in T} c_{t} x_{t} \\
\text { subject to } & \sum_{t \in T} \alpha_{v t} x_{t} \geqslant 1 \quad \text { for all } v \in V, x_{t} \in\{0,1\} \text { for all } t \in T .
\end{array}
$$

The set $V$ of rows to be covered equals now $K_{j}^{*}$, while the set $T$ of columns for covering the rows equals $P_{j}^{\prime}$. An algorithm similar to the one described for cut sets can also be worked out for path sets. Moreover, the remarks following steps 1-6 above remain still valid for the path set case as well. The details are left to the reader.

\section{Genetic algorithms for the SC}

The algorithm in Section 3 requires to solve a series of set covering instances in order to arrive at the computation of $U_{\mathrm{FK}}$. Clearly, pursuing the exact solution of each one, would result to prohibitive execution times. As alternative we use a genetic algorithm (GA) to approximately solve each instance (for a related discussion on meta-heuristics efficiency, see Hordijk and Stadler (1998) or Angel and Zissimopoulos (2000)), thereof obtaining an upper bound of the exact value of $U_{\mathrm{FK}}$. In a recent paper, Beasley and Chu (1996) introduced a GA for SC, which is capable of producing high quality solutions. In our numerical calculations we used the same algorithm with some modifications that we shall discuss later on.

The idea of GA's is based on the evolutionary process of biological organisms in nature. They use a population-based sampling scheme with successive populations biased towards regions where fit solutions (that is, solutions resulting at "desirable" values of a specific objective function) tend to cluster. In general, a GA sets off by taking an initial population of individuals (usually randomly selected) and applies genetic-like operations to produce the "future" generations. Solutions which are more fit, according to the objective function, are more likely to be selected, so that the search tends to regions where optimal solutions are located. After recoding each individual in the population into a string (chromosome) which represents a possible solution, highly fit individuals are participating in a reproduction procedure by exchanging pieces of their genetic information. The main reproduction mechanism in use is a crossover procedure that gives rise to new "offspring" solutions (children) that share some characteristics fetched from both parents. Very often, a further procedure (mutation) is applied after crossover, which alters some bits in the string.

The reproduction mechanism gives one or two offspring solutions that can either replace the whole population (generational approach) or the less fit individuals (steady-state approach). This cycle of evaluation-selection-reproduction is repeated until an appealing solution is reached. The basic steps 


\begin{tabular}{|c|c|c|c|c|c|c|c|}
1 & 2 & 3 & 4 & 5 & $\ldots$ & $\mathrm{n}-1$ & $\mathrm{n}$ \\
\hline 0 & 1 & 1 & 1 & 0 & $\ldots$ & 0 & 1 \\
\hline
\end{tabular}

Fig. 1. Binary representation of a chromosome (solution).

of the GA may be formally stated in the form of an algorithm as follows:

Randomly generate an initial population

Evaluate fitness of each individual in the population (in terms of a pre-specified objective function) Repeat

Select parents from the population that will be used for reproduction,

Apply crossover procedure to the parents to produce children

Perform mutation on children

Evaluate fitness of the children

Replace some or all of the population by children

Until good enough solution has been found

As mentioned before, the first step before applying a GA, is to decide on a suitable representation scheme for encoding the population. For the SC, the usual 0-1 binary string representation is an obvious choice, since it can directly describe the underlying $0-1$ integer variables $\boldsymbol{x}_{t}, t \in T$. Thus, each individual is encoded as an $n$-bit binary string, where $n$ is the number of columns in the SC (see Fig. 1). A value of 1 at the $i$ th bit implies that column $i$ belongs to the solution.

The GA is launched by producing $N$ random individuals as the initial population. Next, the fitness of each individual $i$ is calculated as $\sum_{j=1}^{n} c_{j} b_{i j}$, where $b_{i j}$ is the value of $j$ th bit (column) in the string corresponding to the $i$ th chromosome (child) and $c_{j}$ is the cost of bit (column) $j$.

The crossover mechanism used in the Beasley and Chu (1996) algorithm is the generalized fitness-based crossover, which produces only one child, by taking into account the structures and the relative fitnesses of the two parent solutions. The selection of the two parents is performed according to binary tournament selection. The child that is produced is made feasible according to a heuristic feasibility operator, which initially identifies all uncovered rows and then incorporates additional columns so that all rows are covered. The (feasible) child generated by this mechanism, replaces a randomly chosen member of the population (provided that it is not identical to any other member in it).

In a previous work (Tsitmidelis et al., 2002), a variation of Beasley and Chu's GA has been developed, leading to better results for set covering instances when used for reliability optimization problems. This algorithm has been employed in the present paper as well for carrying out our numerical calculations. The main differences of the modified algorithm (as compared to the original one) may be summarized as follows:

- The crossover mechanism we use to produce two children instead of one. The first child is given birth by the aid of the crossover mechanism of Beasley and Chu according to the relative fitnesses of the two parents. The second one is generated by applying a logical AND function on both parents. The best of the two children is incorporated in the new generation.

- The child that is produced from the crossover mechanism replaces the worst solution in the population at use (instead of the randomly chosen solution used in the original algorithm). 


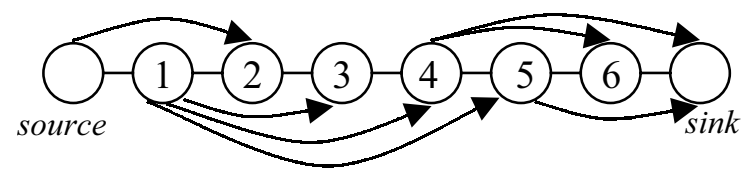

Fig. 2. Example of consecutively connected system with six components.

\section{Numerical results}

In our numerical studies, we commence each time with an initial population of 100 chromosomes (solutions), and the GA is terminated when 1000 nonduplicate children are produced. The mutation procedure selects (at random) five bits and flips their value. For our experiment we used a Pentium III/600 MHz.

Two types of problems were treated. In the first we considered the class of consecutively connected systems and performed repeated calculations selecting several system sizes, component reliabilities and transmission capabilities. In the second we generated the system structure at random by selecting the cut sets among the power set of $\boldsymbol{I}$ so that the resulting structure consists a coherent (monotone) system.

The consecutively connected systems were introduced by Shanthikumar (1987) and can be used to model spacecraft relay stations, telecommunication and oil pipeline systems, vacuum systems in accelerators, etc. Such a system consists of a source $(0), n$ components $\{1, \ldots, n\}$ and a sink $(n+1)$. The source is connected to $\left\{1, \ldots, k_{0}\right\}$ and the component $j(1 \leqslant j \leqslant n)$ is connected to $\left\{j+1, \ldots, j+k_{j}\right\}$ by arcs (integers $k_{j} \geqslant 1,0 \leqslant j \leqslant n$, are called transmission capabilities). The source, sink, and arcs are perfect and the $n$ components are failure prone with failure probabilities $q_{i}=1-p_{i}, i=1,2, \ldots, n$. The system functions if there is a path from the source to the sink through functioning components. In the special case $k_{j}=\min (k, n+1-j), 0 \leqslant j \leqslant n$, the system reduces to the well-known consecutive- $k$-out-of- $n: F$ system (for a review see Chao et al., 1995). Shanthikumar (1987) gave also a recursive scheme for evaluating the exact system reliability.

An example of consecutively connected systems is shown in Fig. 2. In this case we have $n=$ $6, k_{0}=k_{5}=2, k_{1}=4, k_{2}=k_{3}=k_{6}=1, k_{4}=3$, while the family of minimal cut sets is $C=$ $\{(1,2),(1,3),(1,4),(1,5)\}$. If the survival probabilities are given by $p_{1}=p_{2}=p_{3}=0.98$, $p_{4}=p_{5}=p_{6}=0.99$, then the exact reliability $R$ can be evaluated by the Shanthikumar's algorithm as $R=0.99891792$. The lower bound $L_{\mathrm{EP}}$ and upper bound $U_{\mathrm{FK}}$ calculated by our genetic algorithm approach were 0.9989004 and 0.9989183 , respectively.

Our numerical experimentation with consecutively connected systems is displayed in Table 1. Several system sizes $n$, component reliabilities $p_{i}$ and transmission capabilities $k_{i}$ were treated. In Table 1, we have registered the values of $n$, the range of the $p_{i}$ 's and the total number $N$ of cut sets contained in the resulting system. Besides the Esary and Proschan lower bound $L_{\mathrm{EP}}$ and the $\mathrm{Fu}$ and Koutras upper bound $U_{\mathrm{FK}}$, we have also included (to facilitate the comparison of the provided approximation) the reliability estimate $R_{E}=\left(U_{\mathrm{FK}}+L_{\mathrm{EP}}\right) / 2$ and the exact reliability value $R$. A direct inspection of the relative error column (which was computed as $\left.\left(U_{\mathrm{FK}}-L_{\mathrm{EP}}\right) / 2 L_{\mathrm{EP}}\right)$ reveals that, our algorithm leads to quite tight intervals for system's reliability at very reasonable computer times.

The same remarks apply for Table 2, where the system structure was randomly generated; note though that for these cases the exact reliability value cannot be evaluated unless a complete search 
Table 1

Genetic algorithm-based reliability bounds for consecutively connected systems

\begin{tabular}{lrrllllrr}
\hline$P$ & $N$ & $n$ & \multicolumn{1}{l}{$L_{\mathrm{EP}}$} & \multicolumn{1}{l}{$U_{\mathrm{FK}}$} & \multicolumn{1}{l}{$R_{E}$} & $R$ & Computer time (s) & Relative error \\
\hline $0.85-0.95$ & 26 & 50 & 0,8443318 & 0,8449217 & 0,8446268 & 0,8443938 & 22,07 & $3,49 \mathrm{E}-04$ \\
$0.85-0.95$ & 40 & 100 & 0,8476565 & 0,8476698 & 0,8476632 & 0,8476566 & 102,93 & $7,85 \mathrm{E}-06$ \\
$0.80-0.90$ & 52 & 100 & 0,7854174 & 0,7864784 & 0,7859479 & 0,7854555 & 129,73 & $6,75 \mathrm{E}-04$ \\
$0.60-0.70$ & 80 & 150 & 0,5329428 & 0,5400552 & 0,5364990 & 0,5332648 & 417,89 & $6,67 \mathrm{E}-03$ \\
$0.80-0.90$ & 80 & 150 & 0,7905573 & 0,7908107 & 0,7906840 & 0,7905586 & 404,61 & $1,60 \mathrm{E}-04$ \\
$0.90-0.95$ & 80 & 150 & 0,8975495 & 0,8975580 & 0,8975538 & 0,8975495 & 432,63 & $4,74 \mathrm{E}-06$ \\
$0.60-0.70$ & 36 & 200 & 0,5457909 & 0,5459874 & 0,5458892 & 0,5458336 & 174,19 & $1,80 \mathrm{E}-04$ \\
$0.80-0.90$ & 36 & 200 & 0,7915937 & 0,7915940 & 0,7915939 & 0,7915937 & 176,25 & $1,89 \mathrm{E}-07$ \\
$0.90-0.95$ & 36 & 200 & 0,8976591 & 0,8976591 & 0,8976591 & 0,8976591 & 176,41 & $0,00 \mathrm{E}+00$ \\
$0.60-0.70$ & 99 & 200 & 0,5454149 & 0,5455745 & 0,5454947 & 0,5454165 & 855,13 & $1,46 \mathrm{E}-04$ \\
$0.80-0.90$ & 99 & 200 & 0,7918385 & 0,7918386 & 0,7918386 & 0,7918385 & 867,25 & $6,31 \mathrm{E}-08$ \\
$0.60-0.70$ & 252 & 500 & 0,5449945 & 0,5453491 & 0,5451718 & 0,5449953 & 13751,72 & $3,25 \mathrm{E}-04$ \\
$0.80-0.90$ & 252 & 500 & 0,7917534 & 0,7917546 & 0,7917540 & 0,7917534 & 19961,66 & $7,58 \mathrm{E}-07$ \\
\hline
\end{tabular}

Table 2

Genetic algorithm-based reliability bounds for random systems

\begin{tabular}{lrrlllrl}
\hline$P$ & $N$ & $n$ & \multicolumn{1}{l}{$L_{\mathrm{EP}}$} & \multicolumn{1}{l}{$U_{\mathrm{FK}}$} & \multicolumn{1}{l}{$R_{E}$} & Computer time (s) & Relative error \\
\hline $0.80-0.90$ & 98 & 50 & 0,9626803 & 0,9637253 & 0,9632028 & 36,83 & $5,43 \mathrm{E}-04$ \\
$0.60-0.70$ & 50 & 100 & 0,9952515 & 0,9977028 & 0,9964772 & 55,81 & $1,23 \mathrm{E}-03$ \\
$0.80-0.90$ & 50 & 100 & 0,9971187 & 0,9971570 & 0,9971379 & 38,45 & $1,92 \mathrm{E}-05$ \\
$0.80-0.90$ & 49 & 100 & 0,9422784 & 0,9422919 & 0,9422852 & 19,26 & $7,16 \mathrm{E}-06$ \\
$0.80-0.90$ & 100 & 200 & 0,9997982 & 0,9998102 & 0,9998042 & 149,61 & $6,00 \mathrm{E}-06$ \\
$0.80-0.90$ & 100 & 200 & 0,9479110 & 0,9479530 & 0,9479320 & 140,08 & $2,22 \mathrm{E}-05$ \\
$0.50-0.60$ & 100 & 500 & 0,9999994 & 0,9999995 & 0,9999995 & 833,87 & $5,00 \mathrm{E}-08$ \\
$0.50-0.60$ & 100 & 500 & 0,9991869 & 0,9994975 & 0,9993422 & 578,79 & $1,55 \mathrm{E}-04$ \\
$0.80-0.90$ & 100 & 500 & 0,9980323 & 0,9980953 & 0,9980638 & 159,03 & $3,16 \mathrm{E}-05$ \\
$0.80-0.90$ & 250 & 500 & 0,9630716 & 0,9630755 & 0,9630736 & 342,95 & $2,02 \mathrm{E}-06$ \\
\hline
\end{tabular}

is performed over the $2^{n}$ configurations resulting from the states of the $n$ components (something that would require extraordinary computer times).

\section{Conclusion}

In this paper we presented a constructive approach for computing, by the aid of recently introduced bounds, tight lower and upper estimates for the reliability of a general structure. Our modeling through a series of set covering problems, which can be approximately solved by a genetic algorithm, provides excellent estimates for system's reliability at very reasonable computing times.

Two issues seem to be crucial and need further investigation in the future. The first pertains to whether one could exploit the interrelations between the cut sets of the system to avoid repeating 
the genetic algorithm from scratch each time; our impression is that there is a good chance that the (optimal or almost optimal) solutions derived at a set covering instance may be fruitfully used at the subsequent instances. The second issue is related to the problem of inventing an efficient procedure arriving at the most efficient order of the cut sets so that better upper and lower bounds of system's reliability are obtained. It is clear that, succeeding in the first task would give us the opportunity to reduce considerably the required computational time, while an advance towards the second goal would lead to higher quality estimates of system's reliability.

\section{References}

Afif, M., Hifi, M., Paschos, V., Zissimopoulos, V., 1995. A new efficient heuristic for minimum set covering problem. J. Oper. Res. Soc. 46, 1260-1268.

Angel, E., Zissimopoulos, V., 2000. On the classification of NP-complete problems in terms of their correlation coefficient. Discrete Appl. Math. 99, 261-277.

Balas, E., 1980. Cutting planes from conditional bounds: a new approach to set covering. Math. Program. Study 12, $19-36$.

Barlow, R., Proschan, F., 1975. Statistical Theory of Reliability and Life Testing. Holt, Rinehart and Winston, New York. Beasley, J.E., 1990. A Lagrangian heuristic for set covering problems. Naval Res. Logist. 37, 151-164.

Beasley, J.E., Chu, P.C., 1996. A genetic algorithm for the set covering problem. Eur. J. Oper. Res. 94, 392-404.

Boutsikas, M.V., Koutras, M.V., 2000. Generalized reliability bounds for coherent structures. J. Appl. Probab. 37, 778-794.

Chao, M.T., Fu, J.C., Koutras, M.V., 1995. Survey of the reliability studies of consecutive- $k$-out-of- $n$ : $F$ and related systems. IEEE Trans. Reliab. 44, 120-127.

Esary, J.D., Proschan, F., 1963. Coherent structures of non-identical components. Technometrics 5, 191-209.

Fischer, M.L., Kedia, P., 1990. Optimal solutions of set covering/partitioning problems. Manage. Sci. 36, 674-688.

Fu, J.C., Koutras, M.V., 1995. Reliability bounds for coherent structures with independent components. Statist. Probab. Lett. 22, 137-148.

Hordijk, W., Stadler, P., 1998. Amplitude spectra of fitness landscapes. J. Complex System 1, 39-66.

Johnson, D.S., 1974. Approximation algorithms for combinatorial problems. J. Comput. System Sci. 9, 256-278.

Lovász, L., 1975. On the ratio of optimal integral and fractional covers. Discrete Math. 13, 383-390.

Lund, C., Yannakakis, M., 1994. On the hardness of approximating minimization problems. J. ACM 41, 960-981.

Paschos, V., 1997. A survey of approximately optimal solutions to some covering and packing problems. ACM Comput. Surveys 29, 171-209.

Ross, S.M., 1985. Introduction to Probability Models, 3rd Edition. Academic Press, New York.

Shanthikumar, J.G., 1987. Reliability of systems with consecutive minimal cut sets. IEEE Trans. Reliab. 36, 546-549.

Tsitmidelis, S., Zissimopoulos, V., Koutras, M.V., 2002. Fault tree analysis via set cover model. Evolutionary Methods for Design, Optimization and Control, CIMNE, Barcelona, Spain, pp. 179-183. 